\title{
Assessment of Antioxidant and Antimicrobial Compounds of Volatiles from Leaves, Stems and Flowers of Olives
}

\author{
Dhouha Saidana Naija ${ }^{1 *}$, Samia Ben Mansour Gueddes ${ }^{1}$, Guido Flamini², \\ Hayfa Jabnoun Khiareddine ${ }^{3}$, Mejda Daami Remadi ${ }^{3}$, Fathi Ben Mariem', \\ Wafa Ghariani ${ }^{1}$, Mohamed Braham ${ }^{1}$
}

\author{
${ }^{1}$ Olive Tree Institute, Sousse Station, 4061,14 Ibn Khaldoun, Sousse, Tunisia \\ ${ }^{2}$ Università di Pisa, Dipartimento di Farmacia Via Bonanno 33, 56126 Pisa, Italy \\ ${ }^{3}$ Regional Centre of Research on Horticulture and Organic Agriculture (CRRHAB), \\ University of Sousse, Chott-Mariem, Tunisia
}

Received: 12 March 2020

Accepted: 29 April 2020

\begin{abstract}
Protection of olive cultivars, Olea europaea L., from diseases and the development of more sophisticated control methods are indispensable for a renovated and competitive olive sector. In this context, the volatiles obtained by the main Tunisian oil cultivar Chemlali and both the introduced cultivars Arbequina and Koroneiki were tested for their antimicrobial activity against several dangerous pathogens by diffusion and dilution methods (in 2014). To evaluate the adaptation to biotic stress, the antioxidant potential was additionally evaluated. The volatiles extracted from leaves, stems and flowers of the tested cultivars exhibited interesting antimicrobial and antioxidant activities, reaching in many cases $100 \%$ of inhibition. To identify the bioactive compounds, GC-FID and GC-MS were performed, permitting to identify up to $97.8 \%$ of total compounds. Both non-terpene hydrocarbons and terpenes were present in important proportions among volatiles.
\end{abstract}

Keywords: olive cultivars, antimicrobial activity, antioxidant activity, non-terpene hydrocarbons, terpenes

\section{Introduction}

Tunisia is the main olive producing country in the southern Mediterranean. $34 \%$ of its cultivated land is devoted to olive growing, which extends from the

*e-mail: dhouhasaidana@yahoo.com north to the south of the country. This sector plays, economic, social and environmental roles, contributing to food security, job creation, equilibrium of the commercial balance, preservation of natural resources and limitation of the rural exodus. The olive forest is dominated by the oil cultivars, Chemlali in the center and south of the country, and Chetoui in the north. Chemlali alone occupies $56 \%$ of the olive-growing area and represents $69 \%$ of the total number of olive trees 
[1]. Other varieties have been introduced in the Tunisian olive system, such as Arbequina and Koroneiki, of Spanish and Greek origin respectively, to improve the productivity and to mitigate the fluctuation problem that characterizes the local varieties. To maintain satisfying productivity and defend the Tunisian position in the world, the olive tree must be well protected from microbes that may have adverse effects on final yield. Nevertheless in the Mediterranean region, olive production is affected by several diseases, Verticillium wilt, caused by Verticillium dahlia Kleb., is currently the most devastating disease correlated with low yield and high rates of olive tree loss [2]. Fusarium solani causes leaf drop, wilt, and mortality of the olive tree [3]. Pseudomonas is a very dangerous bacterium, Pseudomonas savastanoi and its pathovars savastanoi, fraxini, and nerii provoke a disease characterized by tumorous out growths [4]. The contamination of olive tree by $P$. savastanoi pv. savastanoi causes to hypertrophy of the stems and branches and, rarely, of the leaves and fruits [5]. Similarly, Agrobacterium tumefaciens leads crown gall disease on various plant species, especially olive cultivars, by introducing its T-DNA into the host genome, causing its proliferation and consequently plant tumors. The cited microbes, in the company of many others, have great economic consequences. Since no effective bactericides or fungicides exist, biological control using the naturally occurring antagonistic potential against pathogens is a potentially viable and environmentally friendly alternative [6].

Thus, the aim of the present study is to evaluate the behavior of the principal olive cultivar Chemlali and both the introduced cultivars Arbequina and Koroneiki against many dangerous pathogenic germs and to evaluate their antioxidant capacity to scavenge radicals that could be a consequence of such biotic stress.

\section{Experimental}

\section{Plant Material}

Chemlali, Koroneiki and Arbequina, 35 years old, were cultivated in intensive mode $(6 \times 6)$, in "Menzel El Mhiri", located in Kairouan governorate and Nasrallah delegation ( $35^{\circ} 21^{\prime}$ North $9^{\circ} 49^{\prime}$ East). From each cultivar, fresh leaves, flowers and stems were harvested during the flowering stage, in 2014.

\section{Volatiles Extraction and Analyses}

Volatiles were extracted from the aerial parts of the different cultivars. Fresh leaves, flowers and stems were weighted and crushed and submitted to steam distillation. Obtained samples were conserved at $-16^{\circ} \mathrm{C}$ until tests. The analyses of volatile compounds were performed with GC- FID and GC-MS systems, according to Saidana et al. [7].

\section{Antimicrobial Activities}

The bacterial strains investigated were: Pseudomonas savastanoipvsavastanoi EW2009; Agrobacterium tumefaciens C58; Pseudomonas aureofaciens NCPPB 3335, Burkholderia glathei LMG 14190T (U96935); Bacillus pumilus QST2808.

While fungi strain were Verticillium dahlia MB196942, Botrytis cinerea TAX: 40559, Fusarium solani (Mar.) Sacc. 1881, Penicilliu mitalicum MB162660, Fusarium oxysporum f. sp. Lycopersici MB 416243. The inhibition zones, MIC, MBC and MFC were determined according to Saidana et al. [7, 8].

\section{Antioxidant Activity}

$\mathrm{DPPH}$ and $\mathrm{ABTS}^{\bullet+}$ scavenging activities were performed according to Saidana et al. [9, 10].

\section{Statistical Analysis}

Statistical comparisons of the different parameters were performed with SPSS version 20. Analyses of oneway ANOVA, were followed by means comparisons $(\mathrm{P}=0.05)$ and Tukey test.

\section{Results}

\section{Volatiles Content}

Volatiles yield in Chemlali, Koroneiki and Arbequina leaves, stems and flowers varied significantly from 0.01 to $0.024 \%$.

The highest yield of essential oils was found in the flowers of all the cultivars. Compared to the other flowers, those of Chemlali showed the highest yield, reaching $0.024 \%$.

Similarly, Chemlali appeared to be the richest in volatiles in all its organs, followed by Koroneiki then Arbequina. Indeed, the contents of volatiles were 0.019 , 0.012 and $0.010 \%$ in the leaves, $0.024,0.017$ and $0.015 \%$ in the flowers and $0.012,0.012$ and $0.010 \%$ in the stems of Chemlali, Koroneiki and Arbequina respectively.

\section{Antibacterial Activity}

The antibacterial activities of the volatiles extracted from Chemlali, Arbequina, Koroneiki leaves, stems and flowers were tested against both pathogenic strains, Pseudomonas savastanoi and Agrobacterium tumefaciens and various soil bacteria, such as Pseudomonas aureofaciens, Burkholderia glathei and Bacillus pumilus.

Leaves of the tested cultivars exhibited an antibacterial activity against $P$. savastanoi, with inhibition diameters of 12,13 and $13.5 \mathrm{~mm}$ for Chemlali, Arbequina and Koroneiki, respectively (Table 1). Flowers exhibited even more interesting 


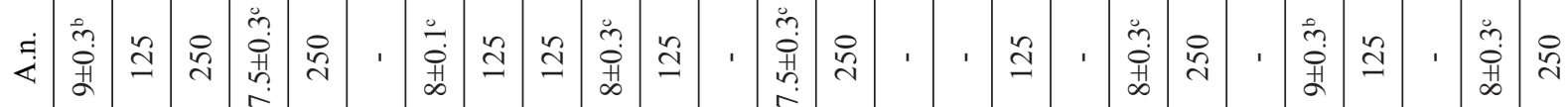

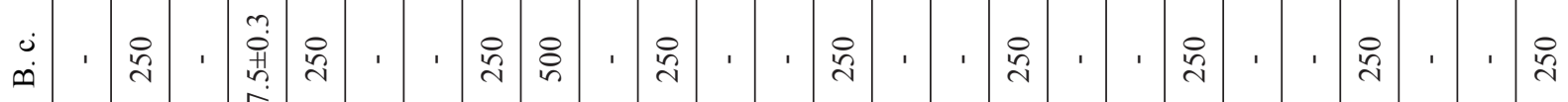

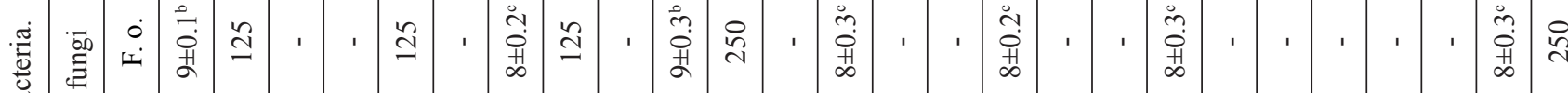

.․․

垔

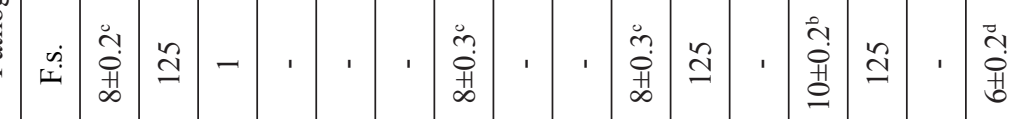

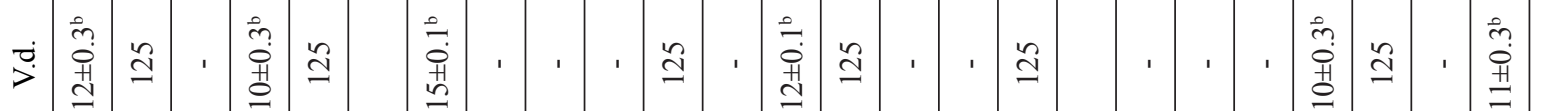

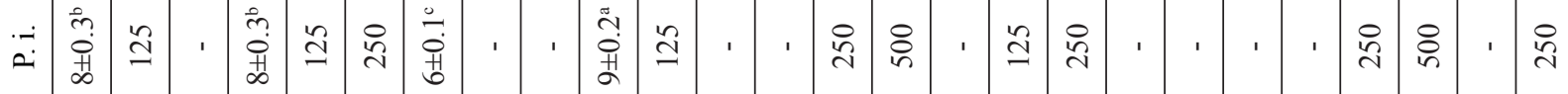

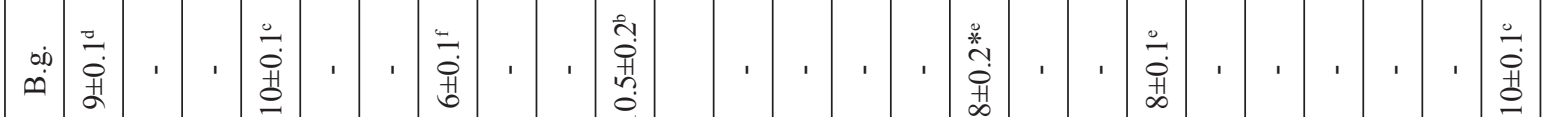

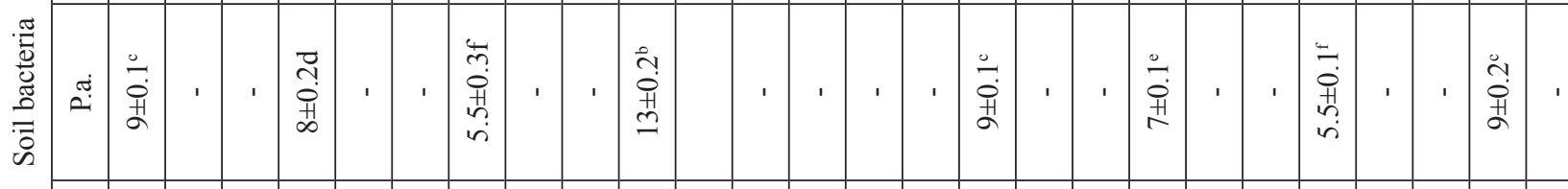

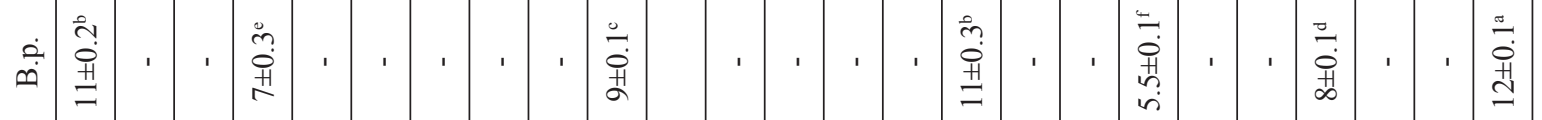

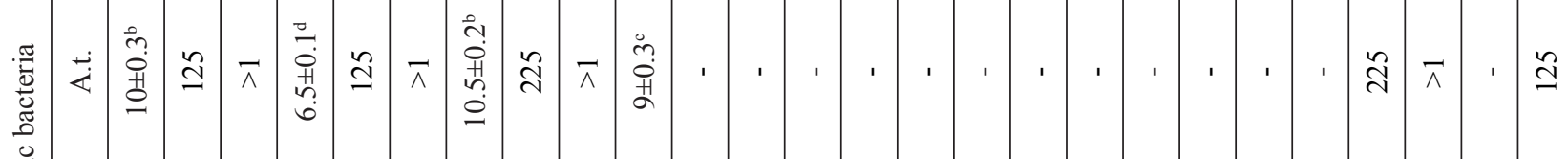

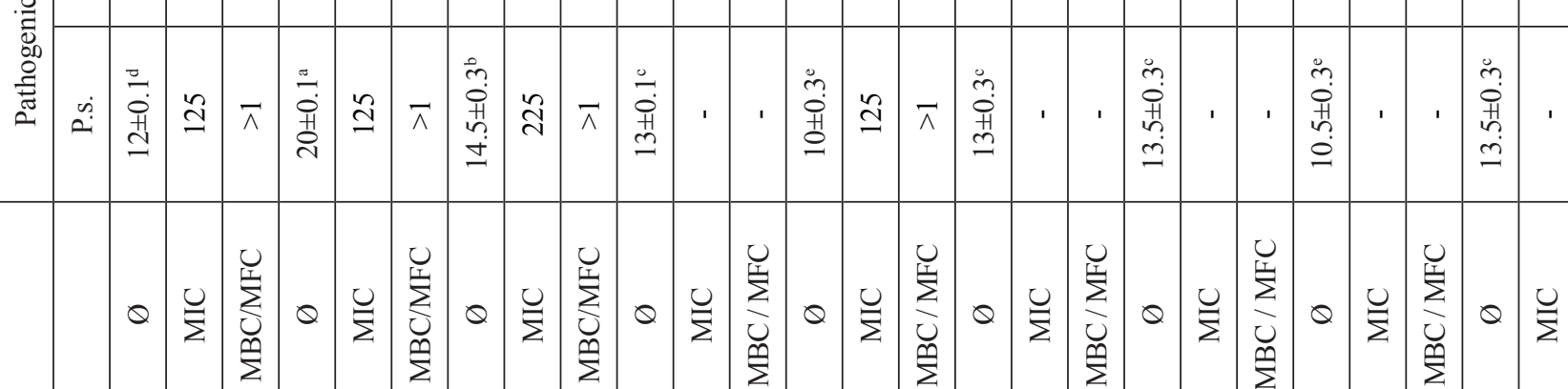

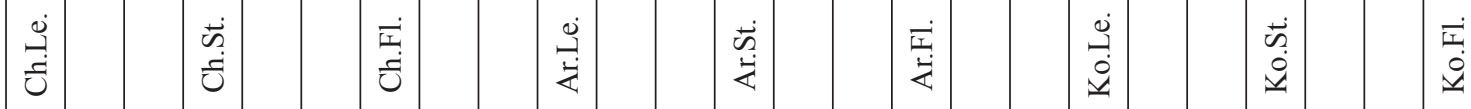




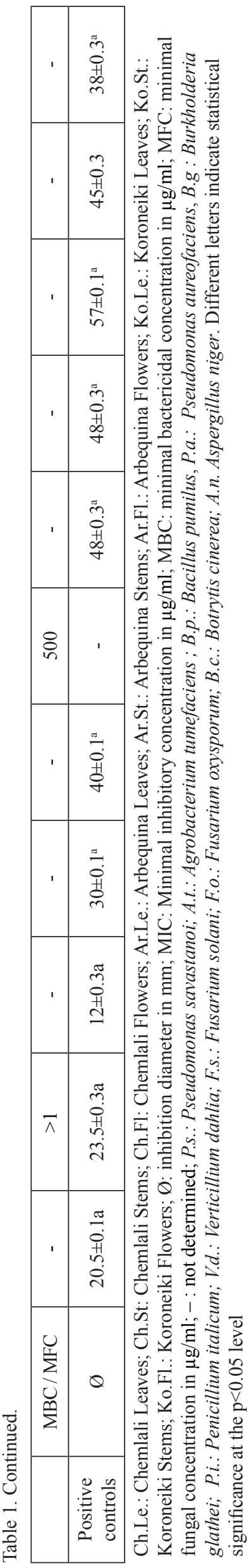

activity, presenting inhibitions zones of 14.5, 13 and $13.5 \mathrm{~mm}$, respectively.

However, stem volatiles of Chemlali showed the best antibacterial activity, which corresponded to the largest inhibition zone, reaching $20 \mathrm{~mm}$. This activity was similar to that of Ampicillin, the antibacterial reference drug. On the contrary, volatiles extracted from stems of Arbequina and Koroneiki presented the least activity against $P$. savastanoi, with an inhibition zone diameter of $10.5 \mathrm{~mm}$. The antibacterial activities of all tested volatiles against $A$. tumefaciens were feeble.

Only the volatiles extracted from all the organs of Chemlali and Arbequina leaves presented diameter inhibition zones varying from 7 to $11 \mathrm{~mm}$ (Table 1). B. pumilus seemed sensitive to flower volatiles of Arbequina and Koroneiki and leaf volatiles of Chemlali, with inhibition zone diameters of 11, 12 and $11 \mathrm{~mm}$. These values were similar to that of Ampicillin, the antibacterial reference drug. P. aureofaciens was moderately sensitive to Arbequina leaf volatiles. While $B$. glathei appeared resistant to all the volatiles. The antibacterial activities of all the samples much smaller than that of Ampicillin against $P$. aureofaciens and $B$. glathei, which presented inhibition zones of 30 and $40 \mathrm{~mm}$, respectively. Stem volatiles of the tested cultivars were inactive against all tested soil bacteria. All Chemlali volatiles extracted from leaves, flowers and stems exhibited an interesting antibacterial activity against $P$. savastanoi and $A$. tumefaciens at quite low concentrations (Table 1). Visible growth inhibitions of both cited bacteria were performed by leaf and stem volatiles of Chemlali at $125 \mu \mathrm{g} / \mathrm{ml}$; whereas flower volatiles were active against these bacteria at $225 \mu \mathrm{g} / \mathrm{ml}$. Bactericidal activities were not determined, being superior to 1 .

Arbequina seemed to be active only through its stem volatiles against $P$. savastanoi, which visible growth was inhibited at $125 \mu \mathrm{g} / \mathrm{ml}$.

While, Koroneiki was active against $A$. tumefaciens through its flower and stem volatiles, with visible growth inhibitions at 125 and $225 \mu \mathrm{g} / \mathrm{ml}$.

\section{Antifungal Activity}

The antifungal activity of the three olive cultivars was tested against six phytopathogenic fungi, Verticillium dahlia, Botrytis cinerea, Fusarium solani, Penicillium italicum, Fusarium oxysporum $f$. $s p$. Lycopersici and Aspergillus niger. According to the results given in Table $1, V$. dahlia appeared to be the most sensitive to volatiles of olive cultivars, especially leaf and flower volatiles of Chemlali, which presented inhibition zones of 12 and $15 \mathrm{~mm}$, respectively. Stem volatiles of Arbequina and flower volatiles of Koroneiki caused inhibition zones of 12 and $11 \mathrm{~mm}$. Leaf, flower and stem volatiles of Chemlali and leaf volatiles of Arbequina exhibited a moderate antifungal activity against $P$. italicum, but interestingly this activity exceeded that 
Table 2. Antioxidant activities (\%) of volatiles extracted from leaves (Le.), stems (St.) and flowers (Fl.) of Chemlali (Ch.), Koroneiki (Ko.) and Arbequina (Ar.) against the radical DPPH at different concentrations (mg/ml).

\begin{tabular}{|c|c|c|c|c|c|c|c|c|c|}
\hline & \multicolumn{9}{|c|}{ Concentration $\mathrm{mg} / \mathrm{ml}$} \\
\hline Samples & 0.125 & 0.25 & 0.5 & 1 & 2 & 4 & 8 & 16 & IC50 \\
\hline Ch.Le. & $\begin{array}{l}27.89 \\
\pm 0.7^{\mathrm{d}}\end{array}$ & $\begin{array}{l}11.22 \\
\pm 0.2^{\mathrm{d}}\end{array}$ & $\begin{array}{l}18.25 \\
\pm 0.6^{\mathrm{c}}\end{array}$ & $\begin{array}{c}37.3 \\
\pm 1.0^{\mathrm{e}}\end{array}$ & $\begin{array}{l}46.82 \\
\pm 3.0^{\mathrm{g}}\end{array}$ & $\begin{array}{l}53.28 \\
\pm 2.5^{\mathrm{g}}\end{array}$ & $\begin{array}{c}68.7 \\
\pm 4.2^{\mathrm{g}}\end{array}$ & $\begin{array}{l}80.15 \\
\pm 1.1^{\mathrm{e}}\end{array}$ & $\begin{array}{c}3 \\
\pm 0.015\end{array}$ \\
\hline Ch.St. & $\begin{array}{l}30.95 \\
\pm 2.1^{\mathrm{d}}\end{array}$ & $\begin{array}{l}30.04 \\
\pm 0.5^{\mathrm{c}}\end{array}$ & $\begin{array}{c}37.3 \\
\pm 1.2^{\mathrm{c}}\end{array}$ & $\begin{array}{l}57.02 \\
\pm 2.1^{\mathrm{bc}}\end{array}$ & $\begin{array}{l}80.61 \\
\pm 3.7^{\mathrm{c}}\end{array}$ & $\begin{array}{l}86.28 \\
\pm 4.1^{\mathrm{bc}}\end{array}$ & $\begin{array}{c}89.9 \\
\pm 1.2^{\mathrm{b}}\end{array}$ & $\begin{array}{l}89.06 \\
\pm 3.6^{\mathrm{b}}\end{array}$ & $\begin{array}{c}0.9 \\
\pm 0.013\end{array}$ \\
\hline Ch.Fl. & $\begin{array}{l}28.23 \\
\pm 0.5^{\mathrm{d}}\end{array}$ & $\begin{array}{l}24.03 \\
\pm 1.2^{\mathrm{c}}\end{array}$ & $\begin{array}{l}38.66 \\
\pm 3.8^{\mathrm{b}}\end{array}$ & $\begin{array}{l}38.88 \\
\pm 2.1^{\mathrm{de}}\end{array}$ & $\begin{array}{l}52.72 \\
\pm 5.1^{\mathrm{e}} \mathrm{f}\end{array}$ & $\begin{array}{l}64.85 \\
\pm 0.1^{\mathrm{f}}\end{array}$ & $\begin{array}{l}74.71 \\
\pm 0.2^{\mathrm{fg}}\end{array}$ & $\begin{array}{l}77.21 \\
\pm 1.9^{\mathrm{f}}\end{array}$ & $\begin{array}{c}1.75 \\
\pm 0.009\end{array}$ \\
\hline Ar.Le. & $\begin{array}{l}52.26 \\
\pm 2.8^{\mathrm{b}}\end{array}$ & $\begin{array}{l}56.12 \\
\pm 2.3^{\mathrm{b}}\end{array}$ & $\begin{array}{l}92.74 \\
\pm 0.9^{\mathrm{a}}\end{array}$ & $\begin{array}{l}94.33 \\
\pm 0.2^{\mathrm{a}}\end{array}$ & $\begin{array}{l}92.29 \\
\pm 0.4^{\mathrm{b}}\end{array}$ & $\begin{array}{l}93.19 \\
\pm 0.4^{\mathrm{ab}}\end{array}$ & $\begin{array}{l}98.54 \\
\pm 4.4^{\mathrm{a}}\end{array}$ & $\begin{array}{l}98.16 \\
\pm 1.2^{\mathrm{a}}\end{array}$ & $\begin{array}{c}0.1 \\
\pm 0.008\end{array}$ \\
\hline Ar.St. & $\begin{array}{l}34.46 \\
\pm 0.5^{\text {cd }}\end{array}$ & $\begin{array}{c}29.5 \\
\pm 0.1^{\mathrm{c}}\end{array}$ & $\begin{array}{l}43.19 \\
\pm 0.2^{\mathrm{c}}\end{array}$ & $\begin{array}{l}68.48 \\
\pm 0.3^{\mathrm{b}}\end{array}$ & $\begin{array}{l}79.02 \\
\pm 1.1^{\mathrm{c}}\end{array}$ & $\begin{array}{l}84.35 \\
\pm 0.7^{\mathrm{c}}\end{array}$ & $\begin{array}{c}84.7 \\
\pm 1.0^{\mathrm{c}}\end{array}$ & $\begin{array}{l}85.51 \\
\pm 2.7^{\mathrm{d}}\end{array}$ & $\begin{array}{c}0.75 \\
\pm 0.003\end{array}$ \\
\hline Ar.Fl. & $\begin{array}{l}27.32 \\
\pm 0.5^{\mathrm{d}}\end{array}$ & $\begin{array}{l}27.77 \\
\pm 0.1^{\mathrm{c}}\end{array}$ & $\begin{array}{l}31.29 \\
\pm 1.5^{\mathrm{bc}}\end{array}$ & $\begin{array}{l}36.05 \\
\pm 2.3^{\mathrm{e}}\end{array}$ & $\begin{array}{l}49.09 \\
\pm 4.3^{\mathrm{fg}}\end{array}$ & $\begin{array}{l}63.71 \\
\pm 0.2^{\mathrm{f}}\end{array}$ & $\begin{array}{l}72.67 \\
\pm 0.3^{\mathrm{g}}\end{array}$ & $\begin{array}{l}77.66 \\
\pm 0.7^{\mathrm{f}}\end{array}$ & $\begin{array}{c}2.4 \\
\pm 0.006\end{array}$ \\
\hline Ko.Le. & $\begin{array}{l}40.92 \\
\pm 0.1^{\mathrm{c}}\end{array}$ & $\begin{array}{l}29.02 \\
\pm 4.0^{c}\end{array}$ & $\begin{array}{l}21.08 \\
\pm 4.0^{\mathrm{c}}\end{array}$ & $\begin{array}{l}37.41 \\
\pm 0.3^{\mathrm{e}}\end{array}$ & $\begin{array}{l}45.01 \\
\pm 3.0^{\mathrm{g}}\end{array}$ & $\begin{array}{l}71.76 \\
\pm 0.9^{\text {ef }}\end{array}$ & $\begin{array}{c}83.21 \\
\pm 0.6^{\text {cde }}\end{array}$ & $\begin{array}{c}86.8 \\
\pm 1.9^{\text {cd }}\end{array}$ & $\begin{array}{c}2.45 \\
\pm 0.014\end{array}$ \\
\hline Ko.St. & $\begin{array}{l}30.83 \\
\pm 0.1^{\mathrm{d}}\end{array}$ & $\begin{array}{l}26.07 \\
\pm 0.1^{\mathrm{c}}\end{array}$ & $\begin{array}{l}39.11 \\
\pm 4.0^{\mathrm{b}}\end{array}$ & $\begin{array}{l}53.17 \\
\pm 1.3^{\text {bc }}\end{array}$ & $\begin{array}{l}73.24 \\
\pm 2.8^{\mathrm{d}}\end{array}$ & $\begin{array}{l}82.32 \\
\pm 1.5^{\mathrm{cd}}\end{array}$ & $\begin{array}{l}85.83 \\
\pm 1.1^{\mathrm{bc}}\end{array}$ & $\begin{array}{l}87.77 \\
\pm 1.5^{\text {bc }}\end{array}$ & $\begin{array}{c}0.8 \\
\pm 0.007\end{array}$ \\
\hline Ko.Fl. & $\begin{array}{c}8.27 \\
\pm 0.2^{\mathrm{e}}\end{array}$ & $\begin{array}{l}25.96 \\
\pm 1.9^{c}\end{array}$ & $\begin{array}{l}41.49 \\
\pm 1.7^{\mathrm{b}}\end{array}$ & $\begin{array}{l}49.77 \\
\pm 1.4^{\text {cde }}\end{array}$ & $\begin{array}{c}6.12 \\
\pm 2.1^{\mathrm{e}}\end{array}$ & $\begin{array}{l}74.48 \\
\pm 3.7^{\mathrm{de}}\end{array}$ & $\begin{array}{l}79.93 \\
\pm 0.1^{\mathrm{de}}\end{array}$ & $\begin{array}{l}81.85 \\
\pm 4.4^{\mathrm{e}}\end{array}$ & $\begin{array}{c}1.5 \\
\pm 0.012\end{array}$ \\
\hline Trolox & $\begin{array}{l}95.39 \\
\pm 0.1^{\mathrm{a}}\end{array}$ & $\begin{array}{l}95.32 \\
\pm 1.2^{\mathrm{a}}\end{array}$ & $\begin{array}{l}94.98 \\
\pm 1.5^{\mathrm{a}}\end{array}$ & $\begin{array}{l}94.45 \\
\pm 2.2^{\mathrm{a}}\end{array}$ & $\begin{array}{c}100 \\
\pm 0.1^{\text {a }}\end{array}$ & $\begin{array}{c}100 \\
\pm 0.0^{\mathrm{a}}\end{array}$ & $\begin{array}{c}100 \\
\pm 0.1^{\text {a }}\end{array}$ & $\begin{array}{c}100 \\
\pm 0.1^{\mathrm{a}}\end{array}$ & \\
\hline
\end{tabular}

Different letters indicate statistical significance at the $\mathrm{p}<0.05$ level for each concentration

of Carbendazime, the antifungal reference drug, which showed no inhibition zone.

Additionally, either F. oxysporum or F. solani were almost resistant to the tested volatiles, with inhibition zones varying between 6 and $10 \mathrm{~mm}$. B. cinerea was the more resistant fungal: only stem volatiles showed an inhibition zone of $7.5 \mathrm{~mm}$. According to the broth dilution method, P. italicum seemed to be the most sensitive against Arbequina and Koroneiki volatiles, where fungicidal activities were noted for the flower and stem volatiles at concentrations varying between 125 to $500 \mu \mathrm{g} / \mathrm{ml}$ (Table 1 ).

Visible growth inhibitions were reached at low concentrations $(125-250 \mu \mathrm{g} / \mathrm{ml})$. Chemlali seemed to be active against this fungus with its stem volatiles, showing a fungicidal activity at $125 \mu \mathrm{g} / \mathrm{ml}$. Additionally, this cultivar exhibited a fungicidal activity against $B$. cinerea at a concentration of $500 \mu \mathrm{g} / \mathrm{ml}$ for its flower volatiles and a visible growth inhibition of this species by its leaf and stem volatiles at $250 \mu \mathrm{g} / \mathrm{ml}$. Only Chemlali leaf volatiles exhibited a fungicidal activity against $F$. solani at a concentration of $1 \mathrm{mg} / \mathrm{ml}$.

Only Arbequina leaf and stem volatiles inhibited a visible growth at $125 \mu \mathrm{g} / \mathrm{ml}$. Similarly, only Chemlali volatiles exhibited a fungicidal activity against $A$. niger at $250 \mu \mathrm{g} / \mathrm{ml}$ in the case of leaves and at $125 \mu \mathrm{g} / \mathrm{ml}$ in the case of flower volatiles. All the tested volatiles exhibited a visible growth inhibition against $A$. niger, at concentrations varying from 125 to $250 \mu \mathrm{g} / \mathrm{ml}$. No fungicidal activity was registered for all the volatiles against $F$. oxysporum $f$. sp. lycopersici, which appeared to be the most resistant species.

However, its visible growth was inhibited by all the volatiles of Chemlali, those of leaves of Arbequina and flowers of Koroneiki.

\section{Antioxidant Activity}

\section{DPPH Scavenging Activity}

The scavenging activity of Chemlali, Koroneiki and Arbequina volatiles, tested at different concentrations and compared with Trolox, is summarized in Table 2.

All the volatiles extracted from the cultivar organs were active against the DPPH radical, and showed a very good antioxidant action. Indeed, almost over $80 \%$ of free radicals were scavenged by all the samples at $16 \mathrm{mg} / \mathrm{ml}$. The inhibition of DPPH was legible even at lower concentrations.

Leaf volatiles of Arbequina showed an important radical scavenging activity (92.74\%) even at $500 \mu \mathrm{g} / \mathrm{ml}$, comparable to that of Trolox at the same concentration. Arbequina leaf volatiles maintained their action even at very low concentrations $(125 \mu \mathrm{g} / \mathrm{ml})$. Interestingly, 50\% of DPPH radicals were inhibited at $100 \mu \mathrm{g} / \mathrm{ml}$ (Table 2). Thus, the leaves of Arbequina seemed to be the most active sample in this test. 


\section{ABTS $^{\bullet+}$ Scavenging Activity}

The variation of the percentage inhibition of volatiles from leaves, stems and flowers of Chemlali, Arbequina and Koroneiki as a function of time and concentration is illustrated in Fig. 1. Volatiles extracted from all the olive tree parts exhibited an interesting scavenging capacity starting from the first five minutes of contact with the radical cation ABTS ${ }^{\cdot+}$. This capacity increased gradually with contact time. Leaf volatiles of Chemlali, Arbequina, Koroneiki seemed to be very active against $\mathrm{ABTS}^{+}$, with $87.5,100$ and $94.85 \%$ of radicals scavenged at a concentration of $16 \mathrm{mg} / \mathrm{ml}$. Leaf volatiles of Arbequina were the most active ones, scavenging the totality of radicals at only $1.0 \mathrm{mg} / \mathrm{ml}$ and in a short time. Chemlali and Koroneiki exhibited a time depending activity, which became more important over time. The inhibitions of $50 \%$ of radicals by leaf volatiles were respectively reached after $30 \mathrm{~min}$ of contact at the concentrations of 1.81, 0.316 and $0.18 \mathrm{mg} / \mathrm{ml}$ for Chemlali, Arbequina and Koroneiki, respectively.
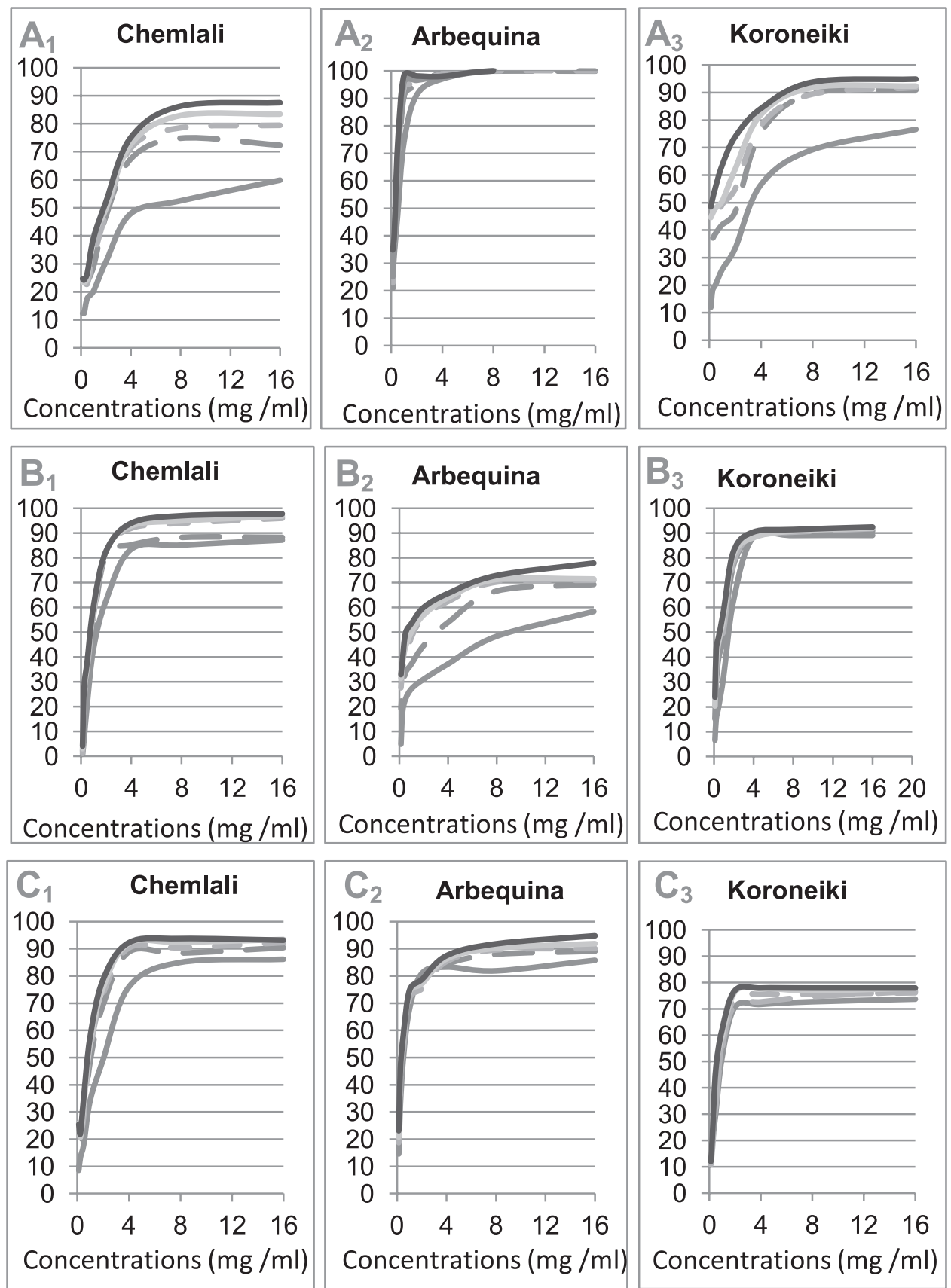

$5 \mathrm{~min}=10 \mathrm{~min}- \pm 15 \mathrm{~min}$

$20 \mathrm{~min} 30 \mathrm{~min}$

Fig. 1. Antioxidant activities (\%) of volatiles extracted from leaves (A1, A2, A3), stems (B1, B2, B3) and flowers (C1, C2, C3) of Chemlali, Arbequina and Koroneiki against the radical cation $\mathrm{ABTS}^{++}$. 
Table 3. Radical cation scavenging activity of Chemlali (Ch.), Arbequina (Ar.) and Koroneiki (Ko.) volatiles extracted from leaves (Le.), stems (St.) and flowers (fl.), expressed as Trolox equivalent after $30 \mathrm{~min}$ of initial mixing and as $50 \%$ of inhibition.

\begin{tabular}{|c|c|c|c|c|c|c|c|c|c|}
\hline & \multicolumn{8}{|c|}{ Concentration $\mathrm{mg} / \mathrm{ml}$} & \multirow[b]{2}{*}{ IC50 } \\
\hline Samples & 0.125 & 0.25 & 0.5 & 1 & 2 & 4 & 8 & 16 & \\
\hline Ch.Le. & $\begin{array}{c}0.60 \\
\pm 0.1^{\mathrm{c}}\end{array}$ & $\begin{array}{c}0.59 \\
\pm 0.1^{\mathrm{cd}}\end{array}$ & $\begin{array}{c}0.65 \\
\pm 0.1^{\mathrm{e}}\end{array}$ & $\begin{array}{c}0.95 \\
\pm 0.0^{\mathrm{d}}\end{array}$ & $\begin{array}{c}1.26 \\
\pm 0.0^{\mathrm{d}}\end{array}$ & $\begin{array}{c}1.81 \\
\pm 0.0^{\mathrm{bc}}\end{array}$ & $\begin{array}{c}2.08 \\
\pm 0.0^{\mathrm{abc}}\end{array}$ & $\begin{array}{c}2.11 \\
\pm 0.0^{\mathrm{bc}}\end{array}$ & $\begin{array}{r}1.810 \\
\pm 0.007\end{array}$ \\
\hline Ch.St. & $\begin{array}{c}0.11 \\
\pm 0.1^{\mathrm{e}}\end{array}$ & $\begin{array}{c}0.68 \\
\pm 0.1^{\text {bcd }}\end{array}$ & $\begin{array}{c}0.93 \\
\pm 0.0^{\mathrm{e}}\end{array}$ & $\begin{array}{c}1.46 \\
\pm 0.1^{\mathrm{cd}}\end{array}$ & $\begin{array}{c}1.99 \\
\pm 0.0^{\mathrm{bc}}\end{array}$ & $\begin{array}{c}2.26 \\
\pm 0.1^{\mathrm{a}}\end{array}$ & $\begin{array}{c}2.33 \\
\pm 0.1^{\mathrm{a}}\end{array}$ & $\begin{array}{c}2.35 \\
\pm 0.0^{\mathrm{ab}}\end{array}$ & $\begin{array}{c}0.722 \\
\pm 0.002\end{array}$ \\
\hline Ch.Fl. & $\begin{array}{c}0.62 \\
\pm 0.0^{\mathrm{c}}\end{array}$ & $\begin{array}{c}0.54 \\
\pm 0.0^{\mathrm{d}}\end{array}$ & $\begin{array}{c}0.85 \\
\pm 0.0^{\mathrm{e}}\end{array}$ & $\begin{array}{c}1.39 \\
\pm 0.0^{\text {cd }}\end{array}$ & $\begin{array}{c}1.89 \\
\pm 0.0^{\mathrm{b}}\end{array}$ & $\begin{array}{c}2.22 \\
\pm 0.0^{\mathrm{ab}}\end{array}$ & $\begin{array}{c}2.25 \\
\pm 0.0^{\mathrm{ab}}\end{array}$ & $\begin{array}{c}2.24 \\
\pm 0.0^{\mathrm{ab}}\end{array}$ & $\begin{array}{c}0.784 \\
\pm 0.027\end{array}$ \\
\hline Ar.Le. & $\begin{array}{c}0.85 \\
\pm 0.0^{\mathrm{b}}\end{array}$ & $\begin{array}{c}1.00 \\
\pm 0.1^{\mathrm{ab}}\end{array}$ & $\begin{array}{c}1.75 \\
\pm 0.0^{\mathrm{a}}\end{array}$ & $\begin{array}{l}2.36 \\
\pm 0.0^{\mathrm{a}}\end{array}$ & $\begin{array}{l}2.36 \\
\pm 0.0^{\mathrm{a}}\end{array}$ & $\begin{array}{l}2.36 \\
\pm 0.0^{\mathrm{a}}\end{array}$ & $\begin{array}{l}2.40 \\
\pm 0.0^{\mathrm{a}}\end{array}$ & $\begin{array}{c}2.40 \\
\pm 0.0^{\mathrm{a}}\end{array}$ & $\begin{array}{c}0.316 \\
\pm 0.028\end{array}$ \\
\hline Ar.St. & $\begin{array}{c}0.80 \\
\pm 0.0^{\mathrm{b}}\end{array}$ & $\begin{array}{c}0.94 \\
\pm 0.0^{\mathrm{abcd}}\end{array}$ & $\begin{array}{c}1.20 \\
\pm 0.0^{\text {bcd }}\end{array}$ & $\begin{array}{c}1.30 \\
\pm 0.0^{\text {de }}\end{array}$ & $\begin{array}{c}1.45 \\
\pm 1.0^{\text {cd }}\end{array}$ & $\begin{array}{c}1.58 \\
\pm 0.0^{\mathrm{d}}\end{array}$ & $\begin{array}{c}1.76 \\
\pm 0.0^{\mathrm{c}}\end{array}$ & $\begin{array}{c}1.88 \\
\pm 0.7^{\mathrm{c}}\end{array}$ & $\begin{array}{c}0.580 \\
\pm 0.003\end{array}$ \\
\hline Ar.Fl. & $\begin{array}{c}0.57 \\
\pm 0.0^{\mathrm{c}}\end{array}$ & $\begin{array}{c}1.09 \\
\pm 0.1^{\mathrm{ab}}\end{array}$ & $\begin{array}{c}1.41 \\
\pm 0.0^{\mathrm{ab}}\end{array}$ & $\begin{array}{c}1.80 \\
\pm 0.0 \mathrm{~b}\end{array}$ & $\begin{array}{c}1.90 \\
\pm 0.0^{\mathrm{b}}\end{array}$ & $\begin{array}{c}2.11 \\
\pm 0.0^{\mathrm{ab}}\end{array}$ & $\begin{array}{c}2.21 \\
\pm 0.1^{\mathrm{ab}}\end{array}$ & $\begin{array}{c}2.28 \\
\pm 0.0^{\mathrm{ab}}\end{array}$ & $\begin{array}{c}0.310 \\
\pm 0.010\end{array}$ \\
\hline Ko.Le. & $\begin{array}{c}1.17 \\
\pm 0.0^{\mathrm{a}}\end{array}$ & $\begin{array}{c}1.24 \\
\pm 0.2^{\mathrm{a}}\end{array}$ & $\begin{array}{c}1.35 \\
\pm 0.1^{\mathrm{abc}}\end{array}$ & $\begin{array}{c}1.53 \\
\pm 0.0^{\mathrm{cd}}\end{array}$ & $\begin{array}{c}1.78 \\
\pm 0.1^{\mathrm{bc}}\end{array}$ & $\begin{array}{c}2.03 \\
\pm 0.1^{\mathrm{ab}}\end{array}$ & $\begin{array}{c}2.26 \\
\pm 0.0^{\mathrm{ab}}\end{array}$ & $\begin{array}{c}2.28 \\
\pm 0.1^{\mathrm{ab}}\end{array}$ & $\begin{array}{c}0.180 \\
\pm 0.014\end{array}$ \\
\hline Ko.St. & $\begin{array}{c}0.58 \\
\pm 0.2^{\mathrm{c}}\end{array}$ & $\begin{array}{c}1.05 \\
\pm 0.1^{\mathrm{ab}}\end{array}$ & $\begin{array}{c}1.17 \\
\pm 0.1^{\text {bcd }}\end{array}$ & $\begin{array}{c}1.42 \\
\pm 0.1^{\mathrm{cd}}\end{array}$ & $\begin{array}{c}1.99 \\
\pm 0.0^{\mathrm{bc}}\end{array}$ & $\begin{array}{c}2.17 \\
\pm 0.0^{\mathrm{ab}}\end{array}$ & $\begin{array}{c}2.20 \\
\pm 0.1^{\mathrm{ab}}\end{array}$ & $\begin{array}{c}2.22 \\
\pm 0.0^{\mathrm{ab}}\end{array}$ & $\begin{array}{c}0.513 \\
\pm 0.007\end{array}$ \\
\hline Ko.Fl. & $\begin{array}{c}0.30 \\
\pm 0.0^{\mathrm{d}}\end{array}$ & $\begin{array}{c}0.55 \\
\pm 0.1^{\mathrm{d}}\end{array}$ & $\begin{array}{c}1.12 \\
\pm 0.0^{\text {bcd }}\end{array}$ & $\begin{array}{c}1.50 \\
\pm 0.0^{\mathrm{cd}}\end{array}$ & $\begin{array}{c}1.85 \\
\pm 0.1^{\mathrm{bc}}\end{array}$ & $\begin{array}{c}1.88 \\
\pm 0.0^{\mathrm{bc}}\end{array}$ & $\begin{array}{c}1.88 \\
\pm 0.0^{\mathrm{bc}}\end{array}$ & $\begin{array}{c}1.88 \\
\pm 0.0^{\mathrm{c}}\end{array}$ & $\begin{array}{c}0.568 \\
\pm 0.005\end{array}$ \\
\hline
\end{tabular}

Different letters indicate statistical significance at the $\mathrm{p}<0.05$ level for each concentration

Koroneiki presented the lowest $\mathrm{IC}_{50}$ after $30 \mathrm{~min}$ of contact (Table 3). Also stem volatiles of the studied cultivars were very active against $\mathrm{ABTS}^{\circ+}$, especially those of Chemlali, which inhibited almost the totality of radicals $\left(\mathrm{IC}_{50}=0.722 \mathrm{mg} / \mathrm{ml}\right)$. Koroneiki and Arbequina seemed to be active even at lower concentrations, with $\mathrm{IC}_{50}$ of 0.513 and $0.580 \mathrm{mg} / \mathrm{ml}$, respectively. Flower volatiles of Chemlali and Arbequina inhibited over 90\% of radicals in almost all time intervals; whereas those of Koroneiki had a slightly weaker activity $(80 \%$ after $30 \mathrm{~min}) . \mathrm{IC}_{50}$ of these cultivars were respectively 0.784 , 0.310 and $0.568 \mathrm{mg} / \mathrm{ml}$ after $30 \mathrm{~min}$ of contact with radicals.

Trolox equivalent antioxidant capacity (TEAC), measured $30 \mathrm{~min}$ after the beginning of the reaction was summarized in Table 3. A TEAC value higher than 1 corresponds to a better antioxidant activity for the tested sample in comparison to Trolox. According to Table 3, Koroneiki leaf volatiles were more active against $\mathrm{ABTS}^{\cdot+}$ than Trolox, even at the lowest tested concentration $(125 \mu \mathrm{g} / \mathrm{ml})$. Arbequina flowers were more active than Trolox at only $250 \mu \mathrm{g} / \mathrm{ml}$, while Chemlali stems and flowers were more active at a concentration of $1 \mathrm{mg} / \mathrm{ml}$. Starting from this concentration $(1 \mathrm{mg} / \mathrm{ml})$, all the olive tree volatiles always exhibited better antioxidant activity than Trolox.

\section{Chemical Composition of Volatiles}

The chemical investigation on the volatiles extracted from different organs of Chemlali, Arbequina and
Koroneiki cultivars permitted to characterize 95.7, 94.9 and $91.7 \%$ of the total compounds in leaves; $80.6,84.4$ and $87.3 \%$ in stems and $95.1,97.8$ and $88.6 \%$ in flowers, respectively (Table 4).

Hydrocarbons appeared to be dominant in all the cultivars, especially in their leaves (49.0, 44.8 and 30.7\% in Chemlali, Arbequina and Koroneiki, respectively). Similarly, terpenes seemed to be the main chemical class in stems and flowers.

Apocarotenes were particularly produced by stems of Chemlali (34.3\%) and flowers of Arbequina (55.4\%) and Koroneiki (44.9\%). Aromatic derivatives were present in relevant amounts in all the cultivars, especially in the leaves (5.4, 16.0 and 14.1\% for Chemlali, Arbequina and Koroneiki, respectively). Aldehydes were particularly detected in flowers $(23.3,18.6$ and14.7\%, respectively).

The major aliphatic compounds were 1-hexadecene (34.4\%, Chemlali leaves), $n$-pentadecane (13.5\%, Arbequina leaves) and $n$-dodecane (10.4\%, Arbequina flowers). Nonanal was the major aldehyde in flowers of Chemlali (12.2\%), Arbequina (10.7\%) and Koroneiki (14.7\%). Dihydroedulan IIA was the most represented apocarotene of Arbequina flowers (18.4\%); while, dihydroedulan IA in Koroneiki flowers (17.8\%) and (E)- $\beta$-damascenone in Chemlali leaves (16.8\%).

(E)-nerolidol and liguloxide were the major oxygenated sesquiterpenes presents in all the cultivar volatiles, reaching the maximum in Koroneiki leaves (13.1\%) and Arbequina stems (11\%). 
Table 4. Chemical composition of volatiles extracted from leaves (Le), stems ( $\mathrm{St}$ ) and flowers (F1) of Chemlali, Koroneiki and Arbequina.

\begin{tabular}{|c|c|c|c|c|c|c|c|c|c|c|}
\hline \multirow[b]{2}{*}{ Constituents (\%) } & \multirow[b]{2}{*}{ l.r.i. } & \multicolumn{3}{|c|}{ Chemlali } & \multicolumn{3}{|c|}{ Arbequina } & \multicolumn{3}{|c|}{ Koroneiki } \\
\hline & & Le & St & Fl & Le & St & Fl & Le & St & Fl \\
\hline 2-methyloctane & 864 & & & & & & & 0.6 & 1.4 & \\
\hline$p$-xylene & 867 & & & & 0.8 & & & 0.2 & 1.5 & \\
\hline$n$-nonane & 900 & & & & 4.1 & 6.5 & & 3.6 & 9.0 & \\
\hline 3-ethyl-1,5-octadiene & 942 & & & & 0.9 & 2.4 & & 1.4 & 2.8 & \\
\hline 1-ethyl-4-methylbenzene & 965 & & & & & & & 0.9 & & \\
\hline phenol & 985 & & & & 1.7 & & & & & \\
\hline 2-methyldecane & 1062 & & 1.8 & & & & & & & \\
\hline linalool & 1101 & & 6.1 & 3.8 & & & 2.3 & 0.3 & & 16.0 \\
\hline nonanal & 1102 & & 3.9 & 12.2 & 0.7 & & 10.7 & 0.6 & & 14.7 \\
\hline camphor & 1145 & & 1.5 & & & & & & & \\
\hline methyl nicotinate & 1148 & & & 15.6 & & & & & & \\
\hline Decane, 5,6-dimethyl- & 1155 & & & & & & & & & \\
\hline Undecane, 2-methyl- & 1167 & & 1.8 & & & & & & & \\
\hline 2-Decanol & 1185 & & & & & & & & & \\
\hline (Z)-3-hexenyl butyrate & 1188 & & & 2.4 & & & 2.1 & & & \\
\hline$\alpha$-terpineol & 1191 & & 3.2 & 21.9 & & & & & & 13.0 \\
\hline methyl salicylate & 1192 & & & & & & 1 & & & \\
\hline$n$-dodecane & 1200 & & & 4.8 & 2.1 & & 10.4 & 0.8 & 1.7 & \\
\hline decanal & 1205 & & & & 0.4 & & & & & \\
\hline trans-piperitol & 1207 & & & & & 2.1 & & & & \\
\hline$\beta$-cyclocitral & 1222 & & 1.4 & & & & & 0.2 & & \\
\hline (E)-2-decenal & 1263 & & & 11.1 & & & 5.3 & & & \\
\hline nonanoic acid & 1275 & & & & & 2.8 & & & & \\
\hline 2,6,11-trimethyldodecane & 1277 & & 3.3 & & & & & & & \\
\hline dihydroedulan IIA & 1285 & & & 8.8 & & & 18.4 & & & 12.7 \\
\hline$p$-cymen-7-ol & 1290 & & & & & & & & & \\
\hline dihydroedulan IA & 1292 & & & 2.3 & & & 7.4 & 0.6 & & 17.8 \\
\hline theaspirane I & 1298 & & & 4.9 & & & 13.6 & & & 4.5 \\
\hline 4-vinylguaiacol & 1313 & & & & 1.3 & & & & & \\
\hline theaspirane II & 1315 & & 2.1 & 3.9 & & & 16.0 & 1.1 & 2.8 & 9.9 \\
\hline methyl 4-formylbenzoate & 1370 & & & & 7.1 & & & & & \\
\hline 3-methyltridecane & 1373 & & & & 2.2 & & & & & \\
\hline (E)- $\beta$-damascenone & 1382 & 16.8 & 3.2 & & 6.1 & & & 4.0 & & \\
\hline 10-acethylmethyl-3-carene & 1389 & 5.5 & 2.7 & & 1.9 & & & 3.1 & 3.1 & \\
\hline 1-tetradecene & 1392 & & 3.1 & & 5.2 & 2.8 & & 1.6 & 3.3 & \\
\hline dihydro- $\gamma$-ionone & 1396 & & 7.4 & & & 10.9 & & & 4.3 & \\
\hline$n$-tetradecane & 1400 & 3.4 & 4.8 & & 12.2 & 7.3 & & 3.6 & 8.9 & \\
\hline (E)- $\beta$-damascone & 1412 & 4.8 & & & 1.2 & & & 1.1 & & \\
\hline trans- $\alpha$-ambrinol & 1414 & & 2.4 & & & 3.2 & & & & \\
\hline
\end{tabular}


Table 4. Continued.

\begin{tabular}{|c|c|c|c|c|c|c|c|c|c|c|}
\hline dihydro- $\alpha$-ionone & 1420 & & & & 3.9 & & & 5.0 & & \\
\hline nerylacetone & 1436 & & 8.0 & & 1.3 & 9.3 & & 3.9 & 10.3 & \\
\hline (E)-geranylacetone & 1456 & & 9.8 & & 3.4 & 8.0 & & 3.4 & 14.2 & \\
\hline (E)- $\beta$-ionone & 1487 & & & & 3.5 & & & 3.4 & 1.8 & \\
\hline 1-pentadecene & 1492 & & & & 2.4 & & & 1.0 & & \\
\hline$n$-pentadecane & 1500 & & 2.3 & & 13.5 & & & & & \\
\hline$(E, E)$ - $\alpha$-farnesene & 1507 & & & & & & & 1.6 & & \\
\hline liguloxide & 1532 & & 9.5 & & & 11.0 & & 2.0 & & \\
\hline dihydroactinidiolide & 1536 & & & & 7.8 & & & 3.5 & & \\
\hline epi-ligulyl oxide & 1551 & & 2.3 & & & 2.7 & & & 5.8 & \\
\hline 4-methylpentadecane & 1556 & & & & 2.2 & & & & & \\
\hline 2-methylpentadecane & 1563 & & & & & & & 6.3 & & \\
\hline$(E)$-nerolidol & 1564 & 9.8 & & & 3.9 & & & 13.1 & 7.8 & \\
\hline (Z)-3-hexenyl benzoate & 1570 & 5.4 & & & 5.1 & & & 11.3 & & \\
\hline hexyl benzoate & 1580 & & & & & & & 1.7 & & \\
\hline caryophyllene oxide & 1582 & & & & & 3.1 & & & & \\
\hline 1-hexadecene & 1593 & 34.3 & & & & 4.9 & & 5.1 & 2.6 & \\
\hline$n$-hexadecane & 1600 & 11.3 & & & & 7.4 & & 6.7 & 6.0 & \\
\hline Valerianol & 1656 & 4.4 & & & & & & & & \\
\hline Hydrocarbons & & 49.0 & 17.1 & 8.2 & 44.8 & 31.3 & 18.4 & 30.7 & 35.7 & \\
\hline Monoterpene hydrocarbons & & 5.5 & 2.7 & & 1.9 & & & 3.1 & 3.1 & \\
\hline Oxygenated monoterpenes & & & 10.8 & 25.7 & & 2.1 & 2.3 & 0.3 & & 29.0 \\
\hline Sesquiterpene hydrocarbons & & & & & & & & 1.6 & & \\
\hline Oxygenated sesquiterpenes & & 14.2 & 11.8 & & 3.9 & 16.8 & & 15.1 & 13.6 & \\
\hline Apocarotenes & & 21.6 & 34.3 & 19.9 & 27.2 & 31.4 & 55.4 & 26.2 & 33.4 & 44.9 \\
\hline Terpenes & & 41.3 & 59.6 & 45.6 & 33.0 & 50.3 & 57.7 & 46.3 & 50.1 & 73.9 \\
\hline Aromatic hydrocarbon & & & & & 0.8 & & & 1.1 & 1.5 & \\
\hline Aromatic esters & & 5.4 & & & 12.2 & & & 13.0 & & \\
\hline Aromatic compounds & & 5.4 & & & 16.0 & & & 14.1 & 1.5 & \\
\hline Aldehydes & & & 3.9 & 23.3 & 1.1 & & 18.6 & 0.6 & & 14.7 \\
\hline Nitrogen compounds & & & & 15.6 & & & & & & \\
\hline Fatty acid and its ester & & & & 2.4 & & 2.8 & 3.1 & & & \\
\hline No aromatic compounds & & & 3.9 & 41.3 & 1.1 & 2.8 & 21.7 & 0.6 & & \\
\hline Total identified compounds & & 95.7 & 80.6 & 95.1 & 94.9 & 84.4 & 97.8 & 91.7 & 87.3 & 88.6 \\
\hline
\end{tabular}

\section{Discussion}

Volatiles from leaves, stems and flowers of Chemlali, Arbequina and Koroneiki cultivars were characterized.

Flowers of all the cultivars were the organs that produced most of the volatiles, with yields reaching $0.024,0.015$ and $0.017 \%$, respectively. Generally, Chemlali produced most volatiles, regardless of the tested organ. Chemlali is of Tunisian origin, while Arbequina and Koroneiki cultivars were introduced for reasons of productivities.

The differences observed for these yields could then be influenced by edaphic and climatic conditions [11], differing in different countries [12]. Additionally, the essential oil content and its composition may vary according to the plant part [13]. To test the effect of 
volatiles extracted from the different organs of the three cultivars, they were tested against the most pathogenic bacteria for the olive tree, Pseudomonas savastanoi and Agrobacterium tumefaciens, by both the diffusion and broth dilution methods. Interaction with some soil bacteria, such as Pseudomonas aureofaciens, Burkholderia glathei and Bacillus pumilus were also noted.

$P$. savastanoi seemed to be more susceptible to the applied olive volatiles than A. tumefaciens, with inhibition zones varying from 10 to $20 \mathrm{~mm}$. All the volatiles seemed to be active against this bacterium, but Chemlali stem volatiles had the best effect. In the case of A. tumefaciens, only volatiles extracted from leaves, flowers and stems of Chemlali and Arbequina leaf volatiles exhibited inhibition zones that reached a maximum of $10.5 \mathrm{~mm}$.

Interaction of olive volatiles with soil bacteria was variable according to the tested microorganism, cultivar and organ. Indeed, B. pumilus seemed to be the most sensitive, with inhibition diameters similar to that of Ampicillin.

The other bacteria, $P$. aureofaciens and B. glathei, presented much smaller inhibition zones $(13$ and $10 \mathrm{~mm}$, respectively) than those registered for the antibacterial reference drug ( 30 and $40 \mathrm{~mm}$, respectively). Chemlali volatiles showed inhibition zones against both bacteria and inhibited their visible growth at quite low concentration $(125 \mu \mathrm{g} / \mathrm{ml})$.

Arbequina and Koroneiki volatiles presented inhibition zones against $P$. savastanoi according to diffusion method, but only stem volatiles of Arbequina inhibited visible growth of this bacterium at $125 \mu \mathrm{g} / \mathrm{ml}$.

Similarly, Koroneiki volatiles, extracted from its flowers and stems, did not show inhibition zones against A. tumefaciens but presented nevertheless a visible inhibition growth at 125 and $225 \mu \mathrm{g} / \mathrm{ml}$, respectively. The negative response of $A$. tumefaciens, when using the diffusion method, may be explained by the high resistance of these Gram-negative bacteria.

Additionally, the diffusion method can greatly vary according to the molecules [14], the organisms tested [15], and the inoculum size. Then, physical and chemical properties of the drugs as well as biological behavior of the bacteria could be put in competition, sometimes with a rather unpredictable outcome [16]. The volatiles were also tested qualitatively and quantitatively against several pathogenic fungi. All the tested volatiles exhibited moderate antifungal activities, with inhibition zones varying from 7.5 to $15 \mathrm{~mm}$. These values were much smaller than those registered for the antifungal reference drug ( 45 to $57 \mathrm{~mm}$ ).

However, all the Chemlali volatiles and Arbequina leaf volatiles exhibited a moderate antifungal activity against $P$. italicum, while the antifungal reference drug presented no activity against this species.

Differently, using the dilution method, almost all the olive volatiles exhibited interesting antifungal activities against the majority of fungi at low doses.
Growth of $F$. solani was totally inhibited by volatiles of Chemlali leaves, while growth of $A$. niger was totally inhibited by volatiles of Chemlali leaves and flowers. Additionally, B. cinerea was totally inhibited by volatiles extracted from Chemlali flowers. Consequently, Chemlali appeared to be the most active cultivar, totally inhibiting the growth of the three pathogenic fungi. $P$. italicum was totally inhibited by stem volatiles of the three olive cultivars, and by Arbequina flower volatiles. Thus P. italicum was the most sensitive species. The antifungal activity of olive volatiles, evaluated by diffusion method, was moderate.

However, this activity was more interesting when micro-dilution method was adopted, with low values of MIC and MFC. This proposed that the size of the inhibition zone does not reflect the real antibacterial efficiency of volatiles, since it is affected by the solubility of the oil, its diffusion in the agar, its evaporation, etc. This point was in agreement with Kim et al. [17] and Cimanga et al. [18] observations.

The essential oil activity is evidently related to the chemical composition of its compounds, their proportions and their interactions each other [19, 20].

Antifungal susceptibility is influenced by the type of medium, the inoculum size, the $\mathrm{pH}$, the temperature and the time of incubation [21]. All tested samples exhibited an interesting antioxidant activity against DPPH radicals, reaching over $80 \%$ inhibition.

The most effective volatiles were those from stems of all the cultivars that inhibited $50 \%$ of radicals in the range $0.75-0.9 \mathrm{mg} / \mathrm{ml}$.

Similarly, an important antioxidant activity was noted for all the volatiles when tested against the cation radicals $\mathrm{ABTS}^{\cdot+}$, reaching $100 \%$ of radical inhibition for some of them. This activity depended on the tested organ, the cultivar and the contact time.

Leaf volatiles of Arbequina appeared to be the most active, scavenging the totality of radicals at only $1 \mathrm{mg} /$ $\mathrm{ml}$ and in a very short time. Chemlali stems and Chemlali and Arbequina flowers were the most active against $\mathrm{ABTS}^{+}$when applied at low concentrations and short time of contact. Trolox equivalent antioxidant capacity measured after $30 \mathrm{~min}$ of contact presented elevated values, demonstrating the powerful antioxidant activity of these volatiles. Awika et al. [22] reported the advantage of $\mathrm{ABTS}^{++}$test over DPPH, as $\mathrm{ABTS}^{++}$test is operable over a wide range of $\mathrm{pH}$, inexpensive and more rapid than the DPPH test. The absorbance of DPPH at $517 \mathrm{~nm}$ is depended on light, oxygen, $\mathrm{pH}$ and type of solvent [23]. Aruoma [24] mentionned that more than one method of antioxidant testing should be used to gain a perceivable indication of antioxidant efficacy of the tested substances. Chemlali, Arbequina and Koroneiki leaf, flower and stem volatiles exhibited interesting antioxidant and antimicrobial activities.

The chemical analyses evidenced the presence of several bioactive compounds. Indeed, all the volatiles contained hydrocarbons in important proportions (up to $49 \%$ ). 1-Hexadecene, the main aliphatic hydrocarbon, 
Table 5. Bioactive compounds identified in Chemlali, Arbequina and Koroneiki volatiles.

\begin{tabular}{|c|c|c|}
\hline Compounds & Biological activity & References \\
\hline \multicolumn{3}{|c|}{ Hydrocarbons } \\
\hline Hexadecene & Antioxidant and antimicrobial activities & {$[26]$} \\
\hline Nonane derivate & Antioxidant and antimicrobial activities & {$[35-36]$} \\
\hline Dodecane & Antioxidant activity & {$[37]$} \\
\hline Trimethyldodecane & Antimicrobial activity & {$[38]$} \\
\hline Pentadecane & Antimicrobial activity & [39] \\
\hline \multicolumn{3}{|l|}{ Terpenes } \\
\hline \multicolumn{3}{|c|}{ Oxygenated Monoterpenes } \\
\hline Linalool & Antioxidant and antimicrobial activities & {$[40-41]$} \\
\hline Terpineol & Antioxidant and antimicrobial activities & {$[42-43]$} \\
\hline \multicolumn{3}{|c|}{ Monoterpenes hydrocarbon } \\
\hline Carene & Antioxidant and antimicrobial activities & {$[44]$} \\
\hline \multicolumn{3}{|c|}{ Oxygenated sesquiterpenes } \\
\hline Nerolidol & Antioxidant and antimicrobial activities & {$[30 ; 45]$} \\
\hline Caryophyllene oxide & Antioxidant and antimicrobial activities & {$[46-47]$} \\
\hline Ligulyl oxide & Antioxidant activity & {$[31]$} \\
\hline \multicolumn{3}{|c|}{ Apocarotenes } \\
\hline$\beta$-Ionone & Antioxidant and antimicrobial activities & [48-49] \\
\hline Dihydroedulan & Antioxidant and antimicrobial activities & {$[50-51]$} \\
\hline Beta-Damascenone & Antioxidant and antimicrobial activities & {$[28 ; 52]$} \\
\hline Geranylacetone & Antioxidant and antimicrobial activities & {$[53]$} \\
\hline Theaspirane & Antioxidant activity & {$[54]$} \\
\hline Hexyl benzoate & Antimicrobial activity & {$[55]$} \\
\hline Methyl 4-formylbenzoate & Antimicrobial activity & {$[56]$} \\
\hline \multicolumn{3}{|c|}{ Aldehyde } \\
\hline Nonanal & Antifungal activity & {$[57]$} \\
\hline \multicolumn{3}{|c|}{ Fatty acid } \\
\hline Nonanoic acid & Antifungal activity & [58] \\
\hline
\end{tabular}

especially in Chemlali leaves $(34.3 \%)$ is known for both antioxidant and antimicrobial activities [25-27]. Also terpenes were well represented among these olive cultivar volatiles, reaching a maximum of $73.9 \%$.

Among apocarotenes, dihydroedulan IIA was particularly detected in Arbequina flowers (18.4\%), while dihydroedulan IA was the major one in Koroneiki flowers (17.8\%). Additionally, many other major bioactive apocarotens, exhibiting antioxidant and antimicrobial activities, were characterized, such as (E)- $\beta$-damascenone (Chemlali leaves, 16.8\%) and (E)-geranylacetone (Koroneiki stems, 14.2\%) [28-29] (Table 5).
Regarding oxygenated sesquiterpenes, the most representative ones were (E)-nerolidol (Koroneiki leaves, 13.1\%) and liguloxide (Arbequina stems, 11.0\%) [30- 31].

Some studies have proved that sometimes the whole volatile extracts have a more powerful biological activity compared to the major component [32 -33]. These authors propose that the compounds present in the greatest proportions were responsible only for a part of the total activity; also, the other components present with smaller amount, contribute to the unregistered activity. As well, a synergistic effect between all components should be considered [34]. 


\section{Conclusion}

Chemlali, Arbequina and Koroneiki volatiles have shown an interesting antibacterial activity against dangerous pathogenic bacteria; in particular, the principal Tunisian cultivar Chemlali exhibited a powerful activity against $P$. savastanoi and $A$. tumefaciens at low concentration $(125 \mu \mathrm{g} / \mathrm{ml})$. Both Chemlali and Arbequina inhibited the visible growth of the majority of the tested fungi at $125 \mu \mathrm{g} / \mathrm{ml}$ through their leaf, stem and flower volatiles and could block completely their growth in many cases. Interestingly, all the tested olive volatiles have an excellent capacity to scavenge radicals. $50 \%$ of radicals were inhibited at $100 \mu \mathrm{g} / \mathrm{ml}$ by the Arbequina leaf volatiles which seemed to be the most active. Many bioactive compounds such as hydrocarbons, oxygenated monoterpenes and apocarotenes, have been identified in the olive cultivar volatiles. These components could contribute to the recorded activities, expected to be related to their stereochemistry, to the proportions in which they are present and to the interactions between them. Further research is required to elucidate the exact mode of action of these active principles. Thus, olive tree volatiles might be a prospective source of alternative antimicrobial and antioxidant agents interesting for a potential use in the biological control or the conservation of food products.

\section{Conflict of Interest}

The authors declare no conflict of interest.

\section{References}

1. MASMOUDI CHARFI C., MSALLEM M., AYADI M., FENDRI M., BEN DHIAB A., BEN ABDALLAH S, OUESLATI A., YAKOUBI S. Caractérisation d'une collection variétale d'oliviers (Olea europaea L.) Efficience de l'utilisation de l'eau. Institut de l'Olivier 1-95, 2017. from IOSfax Website: http://wwwiosfaxagrinettn/annonce/ documentcollectionvarietalepdf

2. LEYVA-PÉREZ M., JIMÉNEZ-RUIZ J., GÓMEZLAMA C.C., VALVERDE-CORREDOR A., BARROSO J.B., LUQUE F., MERCADO-BLANCO J. Tolerance of olive (Olea europaea) cv Frantoio to Verticillium dahliae relies on both basal and pathogen-induced differential transcriptomic responses. New Phytologist, 1-16, 2017.

3. VETTRAINO A.M., SHRESTHA G.P., VANNINI A. First Report of Fusarium solani Causing Wilt of Olea europaea in Nepal. Plant Dis., 93 (2), 200, 2009.

4. PENYALVER R., GARCIA A., FERRER A., BERTOLINI E., LOPEZ M.M. Detection of Pseudomonas savastanoi pv savastanoi in Olive Plants by Enrichment and PCR. App. Env. Microb., 66 (6), 267, 2000.

5. KENNELly M.M., CAZORLA F.M., DE VICENTE A., RAMOS C., SUNDIN G.W. Pseudomonas syringae diseases of fruit trees: progress toward understanding and control. Plant dis., 91, 4, 2007.
6. JIMÉNEZ DÍAZ R.M., OLIVARES-GARCÍA C., LANDA B.B., DEL MAR JIMÉNEZ-GASCO M., NAVAS-CORTÉS J. Region-wide analysis of genetic diversity in Verticillium dahliae populations infecting olive in southern Spain and agricultural factors influencing the distribution and prevalence of vegetative compatibility groups and pathotypes. Phytopathology 101, 304, 2011.

7. SAIDANA D., MAHJOUB M.A., BOUSSAADA O., CHÉRAIF I., CHRIAA J., DAAMI M., MIGHRI Z., HELAL A.N. Chemical composition and antimicrobial activity of volatile compounds of Tamarix boveana (Tamaricaceae). Microb. Res., 163, 445, 2008.

8. SAIDANA D., MAHJOUB S., BOUSSAADA O., CHRIAA J., MAHJOUB M.A., CHÉRAIF I., DAAMI M., MIGHRI, Z., HELAL, A.N. Antibacterial and antifungal activities of the essential oils of two saltcedar species from Tunisia. J. Am. Oil Chem. Soc., 85, 817, 2008.

9. SAIDANA D., BOUSSAADA O., AYED F., MAHJOUB M.A., MIGHRI Z., HELAL A. N. The in vitro free radical scavenging and antifungal activities of the medicinal herb Limonium echioides L growing wild in Tunisia. J. Food Proc. Pres. 37, 533, 2013.

10. SAIDANA D., BOUZIDI A., BOUSSAADA O., HELAL A.N., MAHJOUB M.A., CHÉRAIF I., HELAL A.N., MIGHRI, Z. The antioxidant and free radical scavenging activities of Tamarix boveana and Suaeda fruticosa fractions and related active compound. Eur. Sci. J., 10 (18), 201, 2014 [In French].

11. GILLY G. Perfume plants and essential oils in Grasse: botany, culture, chemistry, production and market, Paris, L'harmattan, 2000.

12. DERWICH E., BENZIANE Z., CHABIR R. Aromatic and medicinal plants of Morocco: Chemical composition of essential oils of Rosmarinus officinalis and Juniperus Phoenicea. Int. J. Appl. Biol.Pharm. Tech., 2 (1), 145, 2011.

13. BRUNETON J. Pharmacognosie: phytochimie, plantes médicinales. $3^{\text {rd }}$ ed, Paris, Lavoisier, 1999 [In French].

14. HAHNE G.B., GOULD R.W. Effects of temperature on biochemical reactions and drug resistance of virulent and avirulent Aeromonas salmonicida. J. Fish. Dis., 5, 329, 1982.

15. MICHEL C., BASSALERT J.F. Influence de la température sur les résultats de l'antibiogramme pratiqué par la méthode de diffusion en ichtyopathologie. Ann. Rech. Vet., 13, 245, 1982 [In French].

16. MICHEL C., BLANC G. Minimal inhibitory concentration methodology in aquaculture: the temperature effect. Aquaculture 196, 311, 2001.

17. KIM J., MARSHALL M.R., WEI C. Antibacterial activity of some essential oil components against five foodborne pathogens. J. Agric. Food Chem., 43, 2839, 1995.

18. CIMANGA K., KAMBU K., TONA L., APERS S., DE BRUYNE T., HERMANS N., TOTTE J., PIETERS L.,VLIETINCK A.J. Correlation between chemical composition and antibacterial activity of essential oils of some aromatic medicinal plants growing in the Democratic Republic of Congo. J. Ethnopharmacol., 79, 213, 2002.

19. MARINO M., BERSANI C., COMI G. Impedance measurements to study the antimicrobial activity of essential oils from Lamiacea and Compositae. Int. J. Food Microbiol., 67, 187, 2001.

20. DELAQUIS STANICH P.J. K., GIRARD B., MAZZA G. Antimicrobial activity of individual and mixed fractions of dill, cilantro, coriander and eucalyptus essential oils. Int. J. Food Microbiol., 74, 101, 2002.

21. GHANNOUM M.A., REX J.H., GALGIANI J.N. Susceptibility testing of fungi: current status of correlation 
of in vitro data with clinical outcome. J. Clin. Microbiol., 34, 489, 1996.

22. AWIKA J.M., ROONEY L.W., WU X., PRIOR R.L. ZEVALLOS L.C. Screening methods to measure antioxidant activity of sorghum (Sorghum bicolor) and sorghum products. J. Agr. Food Chem., 5, 6657, 2003.

23. OZCELIK B., LEE J.H., MIN D.B. Effects of light, oxygen, and $\mathrm{pH}$ on the absorbance of 2,2-diphenyl-1picrylhydrazyl. J. Food Sci., 68, 487, 2003.

24. ARUOMA O.I. Methodological considerations for characterizing potential antioxidant actions of bioactive components in plant foods. Mutat. Res. 9 (20), 523, 2003.

25. KUMAR V., BHATNAGAR A.K., SRIVASTAVA J.N. Antibacterial activity of crude extracts of Spirulina platensis and its structural elucidation of bioactive compound. J. Med. Plants Res., 5 (32), 7043, 2011.

26. MOU Y., MENG J., FU X., WANG X., TIAN J., WANG M., PENG Y., ZHOU L. Antimicrobial and Antioxidant Activities and effect of 1-Hexadecene Addition on palmarumycin C-2 and C-3 Yields in liquid culture of endophytic fungus Berkleasmium sp Dzf12. Molecules, 18 (12), 15587, 2013.

27. MAIMULYANTI A., RESTU PRIHADI A. Chemical composition, phytochemical and antioxidant activity from extract of Etlinger aelatior flower from Indonesia. Journal of Pharmacognosy and Phytochemistry 3(6), 233-238, 2015.

28. ALALAN L., AL-SHAMMAA I., AL-NOURI A.S. Antibacterial activity of essential oil and two of its components extracted from Syrian Inula viscosa against some Syrian gram positive and negative bacteria isolates. J.Chem. Pharm. Res. 7 (12), 857, 2015.

29. BENMEDDOUR T., LAOUER H., AKKAL S., FLAMINI G. Chemical composition and antibacterial activity of essential oil of Launaea lanifera Pau grown in Algerian arid steppes. Asian Pac. J. Trop. Biomed. 5 (11), 960, 2015.

30. KRIST S., BANOVAC D., TABANCA N., WEDGE D.E., GOCHEV V.K., WANNER J., SCHMIDT E., JIROVETZ L. Antimicrobial activity of nerolidol and its derivatives against airborne microbes and further biological activities. Nat. Prod. Commun., 10 (1), 143, 2015.

31. HOSSEINIHASHEMI S.K., ANOOSHEI H., AGHAJANI H., SALEM M.Z.M. Chemical composition and antioxidant activity of extracts from the Inner Bark of Berberis vulgaris Stem. Bioresources 10 (4), 7958, 2016.

32. GILL A.O., DELAQUIS P., RUSSO P., HOLLEY R.A. Evaluation of antilisterial action of cilantro oil on vacuum packed ham. Int J. Food Microbiol., 73, 83, 2002.

33. MOURREY A., CANILLAC N. Anti-Listeria monocytogenes activity of essential oils components of conifers. Food Contr. 13, 289, 2002.

34. BURT S. Essential oils: their antibacterial properties and potential applications in foods. Int. J. Food Microbiol., 94, 223, 2004.

35. HERDAN J.M., CIRA L., GIURGINCA M., IVAN G. Antioxidant activity of some derivatives of 9-thiabicyclo $[3,3,1]$ nonane in rubber and rubber vulcanizates. Polym. Degrad. Stab. 37 (1), 1-5, 1992.

36. PREMALATHA B., BHAKIARAJ D., SELVAM E., GOPALAKRISHNAN M.D. Synthesis, spectral analysis, in vitro microbiological evaluation and antioxidant properties of 2,4-diaryl-3-azabicyclo[3.3.1] nonane- 9-oneO-[2,4,6-tritertiarybutyl-cyclohexa-2,5- dienon-4-yl] oximes as a new class of antimicrobial and antioxidant agents. J. Pharm. Res. 6 (7), 730, 2013.
37. MONTAGNA W., DOBSON R.L. Carcinogenesis: Proceedings of a Symposium on the Biology of Skin Held at the University of Oregon Medical School, 1965. Elsevier Sci. 372, 2017.

38. OLANIPEKUN O.O., OGUNBAYO A.O., NWACHUKWU S.C.U., BELLO R.A. Comparative Study of Microbial Activities and BiodegradationAbilities of Undefined Consortium in Some Hydrocarbon Contaminated Sites in the Niger Delta, Nigeria. J. Env. Prot. 6, 138, 2015.

39. BRUNO F., CASTElli G., MigliazZO A., PIAZZA M., GALANTE A., LO VERDE V., CALDERONE S., NUCATOLO G., FABRIZIO VITALE F. Cytotoxic screening and in vitro evaluation of pentadecane against leishmania infantum promastigotes and amastigotes. J. Parasitol. 101 (6), 701, 2015.

40. DUARTE A., LUÍS A., OLEASTRO M., DOMINGUES F.C. Antioxidant properties of coriander essential oil and linalool and their potential to control Campylobacter spp. Food Contr., 61, 115, 2016.

41. GUNASEELAN S., BALUPILLAI A., GOVINDASAMY K., RAMASAMY K., MUTHUSAMY G., SHANMUGAM M., THANGAIYAN R., ROBERT B.M., NAGARAJAN R.P., PONNIRESAN V.K., RATHINARAJ P. Linalool prevents oxidative stress activated protein kinases in single UVB-exposed human skin cells. PLoS One 3, 1, 2017.

42. PARK S.N., LIM Y.K., FREIRE M.O., CHO E., JIN D., KOOK J.K. Antimicrobial effect of linalool and $\alpha$-terpineol against periodontopathic and cariogenic bacteria. Anaerobe 18 (3), 369, 2012

43. PRAKASH, B., SINGH P., GONI R., KUMAR A., RAINA P., DUBEY N.K. Efficacy of Angelica archangelica essential oil, phenyl ethyl alcohol and $\alpha$ - terpineol against isolated molds from walnut and their antiaflatoxigenic and antioxidant activity. J. Food Sci. Technol. 52 (4), 2220, 2015.

44. MAHBOUBI M., KAZEMPOUR N. Biochemical Activities of Iranian Cymbopogon olivieri (Boiss) Bor. Essential Oil. Indian J. Pharm. Sci. 74 (4), 356, 2012.

45. CHAN W.K., TAN L.T., CHAN K.G., LEE L.H., GOH B.H. Nerolidol: A Sesquiterpene Alcohol with MultiFaceted Pharmacological and Biological Activities. Molecules. 21 (5), 529, 2016.

46. YANG D., MICHEL L., CHAUMONT J.P., MILLETCLERC J. Use of caryophyllene oxide as an antifungal agent in an in vitro experimental model of onychomycosis. Mycopathologia 148(2), 79, 2000.

47. FIDYT K., FIEDOROWICZ A., DAŁA L.S., SZUMNY A. $\beta$-caryophyllene and $ß$-caryophyllene oxide natural compounds of anticancer and analgesic properties. Cancer Med. 5 (10), 3007, 2016

48. BELSITO D., BICKERS D., BRUZE M., CALOW P., GREIM H., HANIFIN J.M., ROGERS A.E., SAURAT J.H., SIPES I.G., TAGAMI H. A toxicologic and dermatologic assessment of ionones when used as fragrance ingredients. Food Chem. Toxico. 45, 130, 2007.

49. ASOKKUMAR S., CHANDRASHEKAR N., SUBRAMANIAN R., SATTU K., PANDI A., SUNDARAM J., THIRUVENGADAM D. Antiproliferative and antioxidant potential of beta-ionone against benzo (a) pyrene-induced lung carcinogenesis in Swiss albino mice. Mol. Cell. Biochem. 363, 335, 2012.

50. SALEHI P., SONBOLI A., ESLAMBOLCHI MOGHADAM S. Essential Oil Composition and 
Antioxidant Activity of Salvia staminea Benth extracts. J. Essent. Oil Bear. Pl. 16 (5), 582, 2013.

51. OZEN T., TELCI I., GUL F., DEMIRTAS I., Chemical analyzes and antioxidant activities of essential oils of four wild Mentha species growing in the Tokat and its districts. Int. J. Chem. Tech. Sayfalar. 46, 2017.

52. BENMEDDOUR T., LAOUER H., AKKAL S., FLAMINI G. Chemical composition and antibacterial activity of essential oil of Launaea lanifera Pau grown in Algerian arid steppes. Asian Pac. J. Trop. Biomed. 5 (11), 960, 2015.

53. BONIKOWSKI R., ŚWITAKOWSKA P., KULA J. Synthesis, odour evaluation and antimicrobial activity of some geranyl acetone and nerolidol analogues. Flav. Fragr. J. 30, 238, 2015.

54. BOUTEBOUHERT H., DIDAOUI L. Study of the chemical composition and antioxidant activity of olive leaves of different varieties (Olea europaea L.) growing in Algeria. Conference: CIPAM 2014, International Congress on Aromatic and Medicinal Plants, Zarzis (Tunis), 2014 [In French].

55. MOJTABA T., REZA G.H., BORZO S., SHIVA N., ESMAEIL S. In vitro Antibacterial and Antifungal Activity of Salvia multicaulis. J. Essent. Oil Bear. Pl. 14 (2), 255, 2011.

56. PELZ K., WIEDMANN-AL-AHMAD M., BOGDAN C., OTTEN J.E. Analysis of the antimicrobial activity of local anaesthetics used for dental analgesia. J. Med. Microb. 57, $88,2008$.

57. KUBICEK C.P., DRUZHININA I.S. Environmental and Microbial relationships. The Mycota, Springer-Verlag Berlin Heidelberg (ed.), 350, 2007.

58. YUN-WOO J., JIN-YOUNG J., IN-KYOUNG L., SIYONG K., BONG-SIK Y. Nonanoic Acid, an Antifungal Compound from Hibiscus syriacus Ggoma. Mycobiology 40 (2), 145, 2012 